\title{
Presentación y objetivos de la Asociación Agroforestal Española (AGFE)
}

\section{Presentation and objectives of the Spanish Agroforestry Association (AGFE)}

\author{
Moreno, G. ${ }^{1 *}$, Mosquera-Losada, M.R. ${ }^{2}$ \\ ${ }^{1}$ Presidente AGFE. Grupo de Investigación Forestal - INDEHESA. Universidad de Extremadura. Plasencia 10600. \\ ${ }^{2}$ Presidente de EURAF. Universidad de Santiago de Compostela. Campus de Lugo. 27002 Lugo \\ *Autor para correspondencia: gmoreno@unex.es
}

\section{Resumen}

La Asociación Agroforestal Española nace oficialmente en 2016, con cuatro finalidades fundamentales: (i) promover la adopción de los sistemas y prácticas agroforestales en España; (ii) trabajar por la aplicación de la PAC de forma adecuada a los sistemas agroforestales; (iii) generar un lugar de encuentro entre investigadores y actores interesados, como son agricultores, ganaderos, selvicultores, empresas y técnicos; (iv) participar de las actividades de la Federación Agroforestal Europea. La actividad se ha centrado fundamentalmente en la mejora de la PAC, logrando que el apoyo a la implementación de nuevos sistemas agroforestales se adopte en varias CC.AA., y que el reglamento llamado ómnibus permita una mejora sensible en la admisibilidad pastos arbolados y arbustivos. Se ha elaborado una publicación sobre Sistemas Agroforestales y PAC para Ambienta, revista oficial del Ministerio de Agricultura. También se ha participado activamente en la elaboración del Informe sobre la Elegibilidad para Pagos Directos de la PAC de los Pastos Leñosos Españoles para el FEGA. Otras actividades a destacar son las participaciones en reuniones mensuales de la EURAF, contribuciones en las Newsletters de la EURAF, traduciéndolas al castellano para su difusión en España y Latinoamérica, participación en varios Grupos Civiles de Diálogo en Bruselas y en el Congreso Agroforestal Europeo (Montpellier 2016 y Nijmegen 2018). Actualmente cuenta con 40 miembros, mayoritariamente del ámbito académico-científico, con tan sólo una empresa y un organismo público. Ahora un objetivo primordial debe ser incorporar más miembros a la asociación, diversificando sus perfiles, y dando más protagonismo a los productores y técnicos.

\section{Summary}

The Spanish Agroforestry Association was officially born in 2016, with four fundamental purposes: (i) to promote the adoption of agroforestry systems and practices in Spain; (ii) work for the application of the CAP in an appropriate way to agroforestry systems; (iii) create a meeting place for researchers and stakeholders, such as farmers, ranchers, foresters, companies and technicians; (iv) participate in the activities of the European Agroforestry Federation. The activity has focused mainly on the improvement of the CAP, achieving that support for the implementation of new agroforestry systems is adopted in several Autonomous Communities, and that the regulation called omnibus allows a sensitive improvement in the admissibility of woodland and shrubby pastures. A publication on Agroforestry Systems and CAP for Ambienta, official journal of the Ministry of Agriculture, has been prepared. It has also actively participated in the preparation of the Report on the Eligibility for Direct Payments of the CAP of the Spanish Woody Pastures for the FEGA. Other activities to be highlighted are the participation in monthly meetings of the EURAF, contributions in the EURAF Newsletters, translating them into Spanish for dissemination in Spain and Latin America, participation in several Civil Dialogue Groups in Brussels and in the European Agroforestry Congress (Montpellier 2016 and Nijmegen 2018). Currently it has 40 members, mostly from the academic-scientific field, with only one company and one public body. Now a primary objective should be to incorporate more members to the association, diversifying their profiles, and giving more prominence to producers and technicians.

Palabras clave: European Federation of Agroforestry, PAC, pastos arbustivos y arbolados, sistemas silvoarables, silvopastoralismo

Keywords: European Federation of Agroforestry, PAC, shrub and woodland pastures, silvoarables systems, silvopastoralism 


\section{Introducción}

Desde el año 2000 viene funcionando en España el Grupo de Trabajo de Sistemas Agroforestales de la Sociedad Española de Ciencias Forestales (SECF), que tuvo su primera reunión científica en Lugo los días 26 y 27 de junio de 2000. Desde entonces ha celebrado reunión científica en 2004 de nuevo en Lugo en el marco del Congreso internacional de Silvopastoralismo, en 2007 en Plasencia (Cáceres), en 2011 en Granada, y en 2016 en Solsona (Lérida). Aunque en todas ellas se ha dado cabida a ponencias y actividades técnicas, lo cierto es que tanto el grupo de trabajo como sus reuniones periódicas se ciñen fundamentalmente al ámbito académico y científico, aunque en alguna de ellas (Granada) la participación de los agricultores ha sido notoria.

Por otro lado, la ciencia agroforestal ha experimentado un avance espectacular en las últimas décadas, con la celebración de tres congresos mundiales (Orlando 2004, Nairobi 2009 y New Delhi 2014), cuatro conferencias europeas (Bruselas en 2012, Cottbus_Alemania en 2014, Montpellier en 2016, Nijmegen en 2018), además de muchos otros eventos mundiales (Silvopastoralism and Sustainable Land Management, Lugo, 2004 y World Congress on Silvo-Pastoral Systems, ÉvoraPortugal 2016) continentales (e.g., conferencias bianuales de la North American Temperate Agroforestry Association desde 1989) y nacionales (reuniones anuales del Farm Woodland Forum en el Reino Unido desde 2001). Además se ha generado un vasto conocimiento científico y multitud de publicaciones en revistas especializadas y libros monográficos (e.g. Mosquera-Losada et al., 2004. Nair et al., 2004; Rigueiro-Rodríguez et al., 2009; Nair and Garrity, 2012).

Sin embargo, la adopción prácticas agroforestales entre propietarios y agricultores es aún escasa en el norte de Europa Europa (Graves et al., 2009; Smith, 2012; Sereke et al., 2016, Mosquera-Losada et al., 2016), quizás con la excepción del sur de Europa. Si bien hay nuevas prácticas agroforestales que se están implantando de forma lenta en países como Francia (Dupraz and Liagre, 2008). En España, la situación tampoco es buena, ni para los sistemas novedosos como los sistemas silvoarables que combinan la plantación de árboles para madera de calidad con cultivos anuales en las calles (e.g. Coello et al., 2018) como para los sistemas tradicionales como la dehesa (Moreno and Pulido, 2009). Debido al escaso avance de las prácticas agroforestales en España y en Europa en su conjunto se considera conveniente impulsar el asociacionismo que vaya más allá del ámbito de la academia, incorporando a los actores que más directamente determinan la implantación y gestión de los sistemas agroforestales, como son los propietarios, agricultores, ganaderos, empresas, y técnicos de la administración. De esta necesidad nace la Asociación Europea Agroforestal en 2012, con estructura de Federación de Asociaciones Nacionales (EURAF: European Agroforesty Federation; www.agroforestry.eu). Del interés en impulsar la adopción de nuevas las prácticas agroforestales en España, además de apoyar las tradicionales, y de la oportunidad de integrarse en la Federación Europea, surge la necesidad de crear una asociación agroforestal en el Estado Español, AGFE, que incorpore socios y abarque objetivos más 
allá del ámbito de la Academia. Por esto nace en 2014, aunque oficialmente aprobada en 2016, la Asociación Agroforestal Española (AGFE), con los siguientes objetivos.

\section{Objetivos}

1.- Promover la adopción de los sistemas y prácticas agroforestales en España;

2.- Trabajar por la aplicación de la PAC de forma adecuada a los sistemas agroforestales, tanto para la aplicación de los pagos de primer pilar como para la implementación de medidas de desarrollo rural (pilar 2);

3.- Generar un lugar de encuentro entre investigadores (ya organizados en torno al grupo de trabajo de la Sistemas Agroforestales de la SECG) y actores interesados, como son agricultores, ganaderos, selvicultores, empresas y técnicos;

4.- Participar de las actividades de la Federación Agroforestal Europea

\section{Actividad}

Durante el periodo 2014-2016 la AGFE ha centrado su actividad fundamentalmente en elaboración de documentos y participación en jornadas relacionadas con la PAC. Dentro del Pilar II, se ha logrado que la medida 8.2 del Pilar II sobre apoyo a la implementación de nuevos sistemas agroforestales se adopte en varias CC.AA. (e.g., Extremadura, Asturias, Galicia), aunque esto no fue el caso para muchas otras. Igualmente se ha logrado dentro del Pilar I mejorar sensiblemente el coeficiente de admisibilidad diseñado para penalizar la presencia de árboles y arbustos en los sistemas silvopastorales. En este sentido se ha elaborado una publicación sobre Sistemas Agroforestales y PAC para Ambienta, revista oficial del Ministerio de Agricultura, Alimentación y Medio Ambiente (Mosquera-Losada et al., 2015). También se participó activamente en la elaboración del Informe sobre la Elegibilidad para Pagos Directos de la PAC de los Pastos Leñosos Españoles para el FEGA (PGEP, 2015). También se ha conseguido que las CC.AA identifiquen y aprueben casos de Prácticas Locales Establecidas en sistemas silvopastorales (pastos arbolados y/o arbustivos), implementada en todas las comunidades autónomas españolas con excepción de Canarias, Baleares, Cataluña y Galicia. Un ejemplo de éxito es el pastoreo con ganado caprino en montes municipales de Extremadura, en los montes de las Sierras del Norte de Extremadura, y los montes de los municipios acogidos a la denominación de origen Quesos de Ibores (la comarca extremeña Ibores- Viluercas). En este caso se consideran superficies admisibles de pastoreo, todos aquellos recintos de pastos arbustivos o arbolados con aprovechamiento ganadero que presenten menos del $50 \%$ de componente herbáceo en su superficie neta tras la aplicación del CAP 2015. Igualmente han sido declaradas Prácticas Locales Establecidas en Asturias las superficies de pastos permanentes declaradas 
en común tanto de propiedad municipal como como privada sobre los que se vienen realizando prácticas tradicionales de aprovechamiento.

En estos dos años AGFE ha participado en la Expo Milan con una actividad de propmoción de la Agroforestería Europea: "Sistemas Agroforestales: Alimentando el planeta y generando energía de forma sostenible”. Otras actividades a destacar han sido la participación en reuniones mensuales de la EURAF, varias contribuciones en las Newsletters de la EURAF (http://www.agroforestry.eu/newsletters), participación en varios Grupos Civiles de Diálogo en Bruselas y reuniones en el Parlamento Europeo. Finalmente señalar la participación activa en la organización de la $3^{\text {a }}$ Conferencia Agroforestal Europea (Montpellier, Mayo 2016).

\section{Retos}

Actualmente cuenta con 40 miembros, mayoritariamente del ámbito académico-científico, con tan sólo una empresa y un organismo público. Una vez creada oficialmente un objetivo primordial debe ser incorporar más miembros a la asociación, diversificando el tipo de miembros, y dando más protagonismo a los productores y técnicos.

Impulsar la página web de la asociación, como sección dentro de la Federación Agroforestal Europea (http://www.agroforestry.eu/countrysection/spain) y la producción periódica de un boletín informativo deben servir para seguir difundiendo las prácticas agroforestales, y a la asociación y sus actividades.

Otro reto importante para AGFE es la participación en la convocatoria de Grupos Operativos para la promoción de todo tipo de prácticas agroforestales. El trabajo activo sobre la reforma intermedia de la PAC así como para que la futura PAC siga dando pasos claros a favor de los sistemas agroforestales será sin duda otra parte importante de la actividad futura de la AGFE.

La inclusión por parte del ministerio español de agricultura de la AGFE como miembro de la Red Rural Nacional permite que los sistemas agroforestales se defiendan en el ámbito de los grupos de trabajo de "Medidas Forestales" y de "Pastos Forestales y Sistemas Silvopastorales" desde finales del 2017, con los que se colaborará para la nueva PAC.

\section{Bibliografía}

Coello, J., Urbán, I., Mosquera-Losada, M.R., 2018. Los sistemas silvoarables modernos en

España. V Reunión del Grupo de Trabajo sobre Sistemas Agroforestales de la Sociedad Española de Ciencias Forestales (SECF). Solsona, 21-22 Junio, 2016.

Dupraz, F., Liagre, F., 2008. Agroforesterie, des arbres et des cultures. France Agricole. Graves, A.R., Burgess, P.J., Liagre, F., Pisanelli, A., Paris, P., Moreno, G.M., Bellido, M., Mayus, M., Postma, M., Schindler, B., Mantzanas, K., Papanastasis, V.P., Dupraz, C., 2009. Farmer perceptions of silvoarable systems in seven European countries. In: 
Rigueiro-Rodríguez, A., McAdam, J.H., Mosquera-Losada, M.R. (eds.), Agroforestry in Europe: Current Status and Future Prospects. Springer, New York.

Moreno, G., Pulido, F., 2009. The Functioning, Management and Persistence of Dehesas. In: Rigueiro-Rodríguez, A., McAdam, J.H., Mosquera-Losada, M.R. (eds.) Agroforestry in Europe: Current Status and Future Prospects. Springer, New-York.

Mosquera-Losada, M.R., McAdam, J., Rigueiro-Rodríguez, A., (eds.), 2009. Silvopastoralism and Sustainable Land Management. CAB International, Wallingford.

Mosquera-Losada, M.R., Moreno, G., Santiago Freijanes, J.J., Ferreiro Domínguez, N., Rigueiro-Rodríguez, A., 2015. Sistemas Agroforestales y PAC. Ambienta 110, 112-124.

Mosquera-Losada, M.R., Santiago Freijanes, J.J., Pisanelli, A., Rois, M., Smith, J., den Herder, M., Moreno, G., Malignier, N., Mirazo, J.R., Lamersdorf, N., Ferreiro Domínguez, N., Balaguer, F., Pantera, A., Rigueiro-Rodríguez, A., Gonzalez-Hernández, P., Fernández-Lorenzo J.L., Romero-Franco, R., Chalmin, A., Garcia de Jalon, S., Garnett, K., Graves, A., Burgess, P.J., 2016. Extent and success of current policy measures to promote agroforestry across Europe. Deliverable 8.23 for EU FP7 Research Project AGFORWARD 613520. 95 pp. Accessed 12 January 2018.

Nair, P.K.R., Garrity, D., 2012. Advances in Agroforestry: Agroforestry - The Future of Global Land Use. Springer Science \& Business Media, Dordrecht. https://doi.org/10.1007/ 978-94-007-4676-3

Nair, P.K.R., Rao, M.R., Buck, L.E. (eds.), 2004. New Vistas in Agroforestry. Advances in Agroforestry: A compendium for 1st world congress. Springer, Dordrecht. https://doi. org/10.1007/978-94-017-2424-1

PGEP, 2015. Informe sobre la elegibilidad para pagos directos de la PAC de los pastos leñosos españoles. Plataforma de ganadería Extensiva y Pastoralismo. http://www. ganaderiaextensiva.org/InformeElegibilidadPastos.pdf Accessed. 12 January 2018.

Rigueiro-Rodríguez, A., McAdam, J., Mosquera-Losada, M.R. (eds.), 2009. Advances in Agroforestry: Agroforestry in Europe: Current Status and Future Prospects. Springer, Dordrecht.

Sereke, F., Dobricki, M., Wilkes, J.J., Kaeser, A., Graves, A.R., Szerencsits, E., Herzog, F., 2016. Swiss farmers don't adopt agroforestry because they fear for their reputation. Agrofor. syst. 90(3), 385-394. https://doi.org/10.1007/s10457-015-9861-3

Smith, J., Pierce, B., Wolke, M.S., 2012. A European perspective for developing modern multifunctional agroforestry systems for sustainable intensification. Renew. Agr. food syst. 27(4), 323-332. https://doi.org/10.1017/S1742170511000597 
